Pacific Journal of Mathematics

RENORMING AND THE THEORY OF PHI-ACCRETIVE
SET-VALUED MAPPINGS 


\title{
RENORMING AND THE THEORY OF PHI-ACCRETIVE SET-VALUED MAPPINGS
}

\author{
David J. Downing and William O. RaY
}

Let $X$ and $Y$ be Banach spaces, $\phi: X \rightarrow Y^{*}$ and $P: X \rightarrow 2^{Y} ; P$ is said to be strongly $\phi$-accretive if there exists $c>0$ so that $(w-v, \phi(x-y))$ $\geq c\|x-y\|^{2}$ whenever $x, y \in X$ and $w \in P x, v \in P y$. Such mappings constitute a simultaneous generalization of monotone mappings (when $Y=X^{*}$ ) and accretive mappings (when $Y=X$ ). By applying a fixed point theorem of $\mathbf{J}$. Caristi, it is shown that if $P$ is strongly $\phi$-accretive in a localized sense and if $Y$ can be appropriately renormed, then, under suitable continuity and range restrictions, $P$ is an open mapping. The results generalize a number of known theorems and indicate a firm connection between the theory of $\phi$-accretive mappings and the renorming characteristics of the space $Y$.

1. Introduction. Let $X$ and $Y$ be Banach spaces with $Y^{*}$ the dual of $Y$, and let $\phi: X \rightarrow Y^{*}$ be a mapping such that

$$
\begin{aligned}
& \qquad \phi(X) \text { is dense in } Y^{*} \\
& \text { for each } x \in X \text { and each } \xi \geq 0,\|\phi(x)\| \leq\|x\| \\
& \text { and } \phi(\xi x)=\xi \phi(x) .
\end{aligned}
$$

A mapping $P$ from $X$ to $Y$ is said to be strongly $\phi$-accretive (e.g. [1] or [14]) if there is a constant $c>0$ such that, for $x, u \in X$

$$
(P x-P u, \phi(x-u)) \geq c\|x-u\|^{2} .
$$

The $\phi$-accretive mappings were introduced in an effort to unify the theories for monotone mappings (when $Y=X^{*}$ ) and for accretive mapping (when $Y=X$ ). While the theorems obtained for the monotone and accretive mappings are similar in character, the methods employed are technically distinct and the goal in the study of $\phi$-accretive operators is to develop a methodology which is applicable to both classes of mappings. Fundamental progress in this direction has been realized by F. E. Browder (e.g. [1]-[4]); one of his basic results in Theorem B below.

Theorem B ([4]). Let $X$ and $Y$ be Banach spaces with $P: X \rightarrow Y a$ strongly $\phi$-accretive mapping. Suppose that one of the following two additional hypotheses holds:

(I) $Y^{*}$ is uniformly convex and $P$ is locally lipschitzian. 
(II) $Y$ and $Y^{*}$ are uniformly convex, $J$ satisfies a Lipschitz condition, $\phi(X)=Y^{*}$, and $P$ satisfies

$$
\|x-u\|^{-1 / 2}\|P x-P u\| \rightarrow 0 \text { as }\|x-u\| \rightarrow 0 .
$$

for each $u$ in $X$.

Then $P(X)=Y$.

Browder's techniques employed a non-convex Bishop-Phelps lemma (Lemma 2 of [3]) and rely upon some fairly deep observations on the geometry of arbitrary Banach spaces. More recently, W. A. Kirk in [13] extended Browder's results to the class of locally $\phi$-accretive mappings under an additional continuity assumption on $P^{-1}: P(X) \rightarrow X$. We note that if $P$ is globally strongly $\phi$-accretive, it follows routinely that $P(X)$ is closed. This need not be the case for mappings of the localized class, hence the assumption on $P^{-1}$. Kirk's innovative approach applied a generalization of the Bishop-Phelps lemma due to I. Ekeland [10]; in particular, he relied on J. Caristi's reformulation of Ekeland's result as a fixed point theorem:

THEOREM C [5]. Let $(M, \rho)$ be a complete metric space, $g$ an arbitrary function from $M$ into $M$, and $\psi$ a lower semicontinuous mapping from $M$ into the non-negative reals. Suppose for each $x \in M$

$$
\rho(x, g(x)) \leq \psi(x)-\psi(g(x)) .
$$

Then $g$ has a fixed point in $M$.

Recently W. Ray [15] has refined Browder's approach and, as a consequence, has obtained sharper results than in [4] or [13]. In this paper we continue the development initiated in [15] and consider set-valued mappings $P: X \rightarrow 2^{Y}$. Moreover, we are able to obtain stronger conclusions than in Theorem B and the subsequent developments of Kirk and Ray. In particular, we show that if $P: X \rightarrow 2^{Y}$ is strongly $\phi$-accretive then, under appropriate assumptions on the mapping $P$ and on the space $Y, P$ is an open mapping; i.e. if $U$ is open in $X, P(U)$ is open in $Y$. Most importantly, however, our results indicate a strong relationship between the mapping theory for $\phi$-accretive operators from $X$ into $Y$ and the renorming characteristics of the space $Y$. This enables us to significantly broaden the class of spaces for which Theorem B remains valid, for example in Theorem $\mathrm{B}(\mathrm{I})$ we need only assume $Y$ is a reflexive Banach space. In $\$ 2$ we state our results, discuss the implied geometric conditions, and derive a number of corollaries; in $\S 3$, we give the proofs.

As a final observation, although all of our results are stated for set-valued mappings, we note that our basic results are new even for single-valued mappings $P: X \rightarrow Y$. 
2. Results Before stating our results we need to introduce some notation and recall some definitions. Let $X$ and $Y$ be Banach spaces. We will be concerned with the following sets:

$$
\begin{aligned}
& \mathscr{B}(X)=\{A \subseteq X: A \text { is closed, bounded and non-empty }\}, \\
& \mathcal{C}(X)=\{A \subseteq X: A \text { is compact and non-empty. }\}
\end{aligned}
$$

Let $\phi: X \rightarrow Y^{*}$ satisfy (1.1) and (1.2).

Definition 2.1. A mapping $P: X \rightarrow \Re(Y)$ is said to be locally strongly $\phi$-accretive if the following condition holds:

For each $x \in X$ there exists $\varepsilon>0$ and a constant $c=c(x)>0$ such that for all $u, v \in B(x ; \varepsilon)$ and all $w \in P u, y \in P v$

$$
(w-y, \phi(u-v)) \geq c\|u-v\|^{2} .
$$

We will say $P: X \rightarrow \mathscr{B}(Y)$ is locally lipschitzian if there exists a constant $L>0$ such that for each $x_{0} \in X$ there is a neighborhood $N\left(x_{0}\right)$ with $H(P u, P v) \leq L\|u-v\|$ for all $u, v \in N\left(x_{0}\right)$; here $H$ represents the Hausdorff metric on $\mathscr{B}(Y)$. (Recall, for any two closed, bounded, nonempty subsets $A$ and $B$,

$$
H(A, B)=\max \left\{\sup _{x \in A} \operatorname{dist}(x, B), \sup _{y \in B} \operatorname{dist}(y, A)\right\}
$$

where dist $(x, B)$ denotes the usual infimum distance between a point and a set.)

Finally, a Banach space $Y$ is said to have a Fréchet differentiable norm if for each $x \in Y$ with $\|x\|=1$

$$
\lim _{\lambda \rightarrow 0} \frac{\|x+\lambda y\|-\|x\|}{\lambda}
$$

exists uniformly for all $y \in Y$ with $\|y\|=1$.

TheOREM 2.1. Let $X$ and $(Y,\|\cdot\|)$ be Banach spaces and suppose $P: X \rightarrow \mathcal{C}(Y)$ is a locally lipschitzian, locally strongly $\phi$-accretive mapping. Suppose, in addition, that $Y$ has an equivalent norm so that $Y$ is Fréchet differentiable and $Y^{*}$ is strictly convex. Then if $U$ is an open subset of $X$, $P(U)$ is an open subset of $Y$.

The next result follows as an immediate corollary.

Corollary 2.1. Let $X, Y$ and $P: X \rightarrow \mathcal{C}(Y)$ be as in Theorem 2.1. If $P(X)$ is closed in $Y$, then $P(X)=Y$. 
Let $J: Y \rightarrow 2^{Y^{*}}$ denote the duality mapping from $Y$ into $2^{Y^{*}}$; i.e., the mapping given by

$$
J(x)=\left\{x^{*} \in Y^{*}:\left\|x^{*}\right\|^{2}=\|x\|^{2}=\left(x, x^{*}\right)\right\}
$$

where $(\cdot, \cdot)$ denotes the generalized duality pairing. If $Y^{*}$ is strictly convex, then, as is well-known, $J$ is single-valued. Moreover a classical theorem of V. Smul'yan $[17,18]$ states that when $Y$ has a single-valued duality mapping, that mapping is norm-to-norm continuous on bounded sets if and only if $Y$ has a Fréchet differentiable norm. Thus Theorem 2.1 (and in particular Corollary 2.1) represent extensions of Theorem B(I) and the subsequent development by Kirk and Ray to a much broader class of spaces, as well as to the case when the mapping is set-valued. (Definition 2.1 varies slightly from the definition of locally strongly $\phi$-accretive in [13] or [15]. If one assumes the definition of [13], Corollary 2.1 is still valid-see \$3-although our proof of Theorem 2.1 does not carry over.)

Particular classes of spaces satisfying the renorming conditions of Theorem 2.1 are generally well-known and we summarize at least part of this information in Proposition D below. As usual, either [6] or [8] are excellent references. Recall that a Banach space $Y$ is weakly compactly generated (WCG) if there exists a weakly compact set $K \subseteq Y$ such that the linear span of $K$ is dense in $Y$. Easy examples of WCG spaces are $c_{0}(\Gamma)$ for any index set $\Gamma$ and any separable space. Thus, in light of Proposition $\mathrm{D}$, any Banach space $Y$ with separable dual satisfies the renorming criteria of Theorem 2.1.

Proposition D (cf. [6, page 160]). Let Y be a Banach space. Suppose either

(i) $Y=c_{0}(\Gamma)$ for some index set $\Gamma$, or

(ii) Both $Y$ and $Y^{*}$ are WCG.

Then $Y$ can be renormed so that $Y^{*}$ is strictly convex and $Y$ has a Fréchet differentiable norm.

The proof of Proposition D essentially consists of showing that in either case $Y$ can be renormed so that $Y^{*}$ is dually locally uniformly convex. (Thus, given a sequence $y_{n}^{*}$ of norm one elements and an element $y_{0}^{*}$ of norm one for which $\frac{1}{2}\left\|y_{n}^{*}+y_{0}^{*}\right\| \rightarrow 1$, we must have $y_{n}^{*} \rightarrow y_{0}^{*}$.) If (i) holds, this is accomplished by appealing to a theorem of Troyanski [19] which shows $l_{1}(\Gamma)$ can be renormed to be locally uniformly convex and then observing this new norm is a dual norm; in case (ii), the result is the John-Zizler renorming theorem [12]. The proof is concluded by noting $Y^{*}$ locally uniformly convex implies $Y^{*}$ is strictly convex and $Y$ has Fréchet differentiable norm (cf. [8, pg. 31-32].) Of course, as a consequence of this last remark, any space $Y$ which can be renormed so that $Y^{*}$ is locally uniformly convex satisfies the assumptions of Theorem 2.1 . 
We have already mentioned one example of WCG spaces with WCG dual. One final class of spaces satisfying (ii) of Proposition D merit special attention.

THEOREM 2.2. Let $X$ and $Y$ be Banach spaces with $Y$ reflexive. Suppose $P: X \rightarrow \Re(Y)$ is a locally lipschitzian, locally strongly $\phi$-accretive mapping with Px convex for each $x \in X$. Then, if $U$ is open in $X, P(U)$ is open in $Y$. In particular, if $P(X)$ is closed, $P(X)=Y$.

We now turn to some immediate implications of the two previous theorems. For the first of these assume that $\phi: X \rightarrow Y^{*}$ satisfies (1.1) and (1.2) and that $c:[0, \infty) \rightarrow[0, \infty)$ is a nonincreasing, continuous function with $\int_{a}^{\infty} c(t) d t=\infty$ for each $a \geq 0$. Theorems 2.1 and 2.2 yield the following improvements of Theorem 4.11 of [1].

COROLlary 2.2. Let $X$ and $Y$ be Banach spaces with $Y$ satisfying the renorming conditions of Theorem 2.1. Let $P: X \rightarrow \mathcal{C}(Y)$ be a locally strongly $\phi$-accretive mapping. Suppose, in addition, $P$ satisifies

$$
\begin{gathered}
\text { for each } x, y \in X \text { and } x \in P x, u \in P y \\
(w-u, \phi(x-y)) \geq c(\max \{\|x\|,\|y\|\})\|x-y\|^{2} .
\end{gathered}
$$

Then $P$ is an open mapping with $P(X)=Y$.

Corollary 2.3. Let $X$ and $Y$ be Banach spaces with $Y$ reflexive. Suppose $P: X \rightarrow \Re(Y)$ is a locally lipschitzian mapping satisfying (2.2) with $P x$ convex for each $x \in X$. Then $P$ is an open mapping with $P(X)=Y$.

We note that Corollary 2.2 (respectively Corollary 2.3 ) follows immediately from Theorem 2.1 (respectively Theorem 2.3) and Theorem 3.3 of [16]. While Browder's original result only deals with mappings from $X$ into $X$; we are able to consider mappings from a space $X$ into a different space $Y$. Thus Corollaries 2.2 and 2.3 represent an extension of Browder's result into the theory of $\phi$-accretive mappings from $X$ into $Y$. Theorem 4.11 of [1] has recently been extended in yet another fashion. W. Ray and A. Walker [16] have shown that any continuous mapping $P: X \rightarrow X$ satisfying $\left(P x-P y, J(x-y) \geq c(\max \{\|x\|,\|y\|\})\|x-y\|^{2}\right.$ is in fact a homeomorphism of $X$ onto $X$. Their approach involves showing $P$ is an open mapping by using standard techniques from the theory of ordinary differential equations; although we retain the locally lipschitzian condition on $P$ our approach is rather more self-contained and avoids any reliance on differential equations.

Our next corollary represents a localized version of some results of Deimling, however we need to place more restrictive conditions on both the space $X$ and the mapping than are required in [7]. 
COROLlaRY 2.4. Let $X$ be a reflexive space with $X^{*}$ strictly convex. Suppose $P: X \rightarrow \Re(X)$ is a locally lipschitzian mapping with $P x$ convex for each $x \in X$. Suppose, in addition, the mapping $P$ satisfies

for each $x_{-} \in X$, there is a neighborhood $N\left(x_{0}\right)$ and a constant $c=c\left(x_{0}\right)>0$ so that for all $u, v \in N\left(x_{0}\right)$ and $w \in P u, y \in P v$ :

$$
(w-y, J(u-v)) \geq c\|u-v\|^{2}
$$

where $J: X \rightarrow X^{*}$ is the duality mapping. If $U$ is open in $X$. then $P(U)$ is open in $Y$.

We add parenthetically that if $X$ and $P: X \rightarrow \mathscr{B}(X)$ satisfy the assumptions of Corollary 2.4 and if $P(X)$ is closed, then $P(X)=Y$. Moreover, if $P: X \rightarrow \mathcal{C}(X)$, we need only assume that $X^{*}$ is strictly convex and that $X$ can be renormed to be Fréchet differentiable; in particular, under this additional assumption on the mapping $P$, any space $X$ with $X^{*}$ locally uniformly convex will suffice in Corollary 2.4. Following [7], we will say a mapping $P: X \rightarrow X$, where $X^{*}$ is strictly convex, is said to be strongly accretive if for each $x, y \in X$

$$
(P x-P y, J(x-y)) \geq \alpha(\|x-y\|)\|x-y\|,
$$

where $\alpha:[0, \infty) \rightarrow[0, \infty)$ is a continuous function with $\alpha(0)=0$ and $\alpha(t)>0$ for $t>0$. If, in addition, we impose the condition that $\liminf _{t \rightarrow 0} \alpha(t) / t>0$ (as in [7, Theorem 1]), then condition (2.4) clearly implies (2.3). Thus any strongly accretive mapping (in the sense of [7]) with $\liminf _{t \rightarrow 0} \alpha(t) / t>0$ will satisfy Corollary 2.4 .

In each of the preceding results we have required the mapping to be locally lipschitzian. As in Theorem B(II), this continuity assumption can be weakened by placing a more restrictive geometric condition on $Y^{*}$. We will also assume $\phi: X \rightarrow Y^{*}$ is a mapping satisfying (1.2) with $\phi(X)=Y^{*}$.

TheORem 2.3. Let $X$ and $Y$ be Banach spaces and let $P: X \rightarrow \Re(Y)$ be a locally strongly $\phi$-accretive mapping with $P x$ convex for each $x \in X$. Suppose P satisfies the condition:

$$
\begin{gathered}
\text { for each } u, v \in X \text { and } y \in P u, \\
\liminf _{t \rightarrow 0} \operatorname{dist}\left(y, P\left(x_{t}\right)\right) /\left\|x_{t}-u\right\|^{1 / 2}=0
\end{gathered}
$$

where $x_{t}=u+t v$. Suppose, in addition, that $Y$ can be renormed so that $Y^{*}$ is strictly convex and so that the duality mapping $J: Y \rightarrow Y^{*}$ is lipschitzian on bounded sets. Then, if $U$ is open in $X, P(U)$ is open in $Y$. 
Condition (2.5), which in the single-valued case reduces to a Hölder condition of order $1 / 2$ along line segments, was introduced in [9] where surjectively results are obtained for locally strongly $\phi$-accretive set-valued mappings which do not necessarily have closed range. However in [9, Theorem 4] it is assumed that both $Y$ and $Y^{*}$ are uniformly convex. If $P: X \rightarrow Y$ is a single-valued mapping then Theorem 2.3, under the assumption that $Y^{*}$ is strictly convex and $J: Y \rightarrow Y^{*}$ is lipschitzian, is implicit in [15].

We should note that any space $Y$ satisfying Theorem 2.3 is superreflexive (and hence reflexive.) This is easily seen by combining a theorem of V. Smul'yan $[17,18]$ with the fundamental result of Per Enflo [11] characterizing those spaces which can be renormed to be uniformly convex.

Since the assumptions on the mapping in Theorem 2.3 are of a local nature, we make one final observation.

Corollary 2.5. Let $X, Y$ and $P: X \rightarrow \Re(Y)$ be as in Theorem 2.3. If $P(X)$ is closed in $Y, P(X)=Y$.

3. Proofs. The proofs of our main results are based on the proofs of Theorems 1 and 2 in [15]. As a preliminary observation, we note that if $(M, \rho)$ is a metric space and $H$ is the Hausdorff metric generated by $\rho$ then, for any $\varepsilon>0$ and any two closed, bounded subsets $A$ and $B \subseteq M$, given $a \in A$ we can choose $b \in B$ so that $\rho(a, b) \leq H(A, B)+\varepsilon$. Moreover, if $A$ and $B$ are compact, we can choose $b \in B$ with $\rho(a, b) \leq$ $H(A, B)$.

Proof of Theorem 2.1. Let $\|\cdot\|_{1}$ be the norm on $Y$ which is Fréchet differentiable with strictly convex dual norm. Then the duality mapping $J$ : $\left(Y,\|\cdot\|_{1}\right) \rightarrow 2^{\left(Y,\|\cdot\|_{1}\right)^{*}}$ is single-valued and norm-to-norm continuous. Notationally, for $A \subseteq Y$, let $V(A ; r)=\{y \in Y$ : $\operatorname{dist}(y, A) \leq r\}$ (where $\left.\operatorname{dist}(y, A)=\inf \left\{\|y-a\|_{1}: a \in A\right\}\right)$ and let $H$ be the Hausdorff metric generated by $\|\cdot\|_{1}$. For any Banach space $E$ and $x \in E$, we will let $B(x ; r)=\{y \in E:\|y-x\| \leq r\}$.

Since $\|\cdot\|_{I}$ is an equivalent norm, $P$ is locally lipschitzian with respect to $H$ and, for each $x \in X, P x$ is compact in $\left(Y,\|\cdot\|_{1}\right)$. Moreover, there exist constants $n, N>0$ so that

$$
n\|\phi(x)\|^{*} \leq\|\phi(x)\|_{1}^{*} \leq N\|\phi(x)\|^{*}
$$

whenever $x \in X$ (here $\|\cdot\|^{*}$ is the dual of the original norm on $Y$ and $\|\cdot\|_{1}^{*}$ is the dual norm of $\|\cdot\|_{1}$.) Define $\tilde{\phi}: X \rightarrow Y^{*}$ by $\tilde{\phi}=\phi / N$. Then

(i) $\tilde{\phi}(X)$ is dense in $\left(Y,\|\cdot\|_{1}\right)^{*}$, 
(ii) for each $x \in X$ and $\xi>0, \tilde{\phi}(\xi x)=\xi \tilde{\phi}(x)$ and

$$
\|\tilde{\phi}(x)\|_{1}^{*}=\frac{1}{N}\|\phi(x)\|_{1}^{*} \leq\|\phi(x)\|^{*} \leq\|x\| .
$$

Let $U \subseteq X$ be open. Fix $x_{0} \in U$ and choose $\varepsilon_{1}>0$ so that $P$ is lipschitzian on $B\left(x_{0} ; 2 \varepsilon_{1}\right)$ - say with constant $L$-and so that $B\left(x_{0} ; 2 \varepsilon_{1}\right)$ $\subseteq U$. Using the facts that $P$ is locally strongly $\phi$-accretive and that $\|\cdot\|_{1}$ is an equivalent norm along with the definition of $\tilde{\phi}$ allows us to select $c=c\left(x_{0}\right)>0$ and $\varepsilon_{2}>0$ so that

$$
\text { if } u, v \in B\left(x_{0} ; 2 \varepsilon_{2}\right) \text {, then for all } w \in P u, z \in P v
$$

$$
(w-z, \tilde{\phi}(u-v)) \geq c\|u-v\|^{2} .
$$

Set $\varepsilon=\min \left\{\varepsilon_{1}, \varepsilon_{2}\right\}$ and $r=c \varepsilon / 2$. It suffices to show $V\left(P x_{0} ; r\right) \subseteq P(U)$, so let $y \in V\left(P x_{0} ; r\right)$ and note

$$
\text { if } x \in B\left(x_{0} ; 2 \varepsilon\right) \text { and } \operatorname{dist}(y, P x) \leq r \text {, then }\left\|x-x_{0}\right\| \leq \varepsilon .
$$

To verify (3.2), let $x$ satisfy these assumptions and suppose $z \in P x$. By (3.1), for each $z_{0} \in P x_{0}$,

$$
c\left\|x-x_{0}\right\|^{2} \leq\left(z-z_{0}, \tilde{\phi}\left(x-x_{0}\right)\right) \leq\left\|z-z_{0}\right\|_{1}\left\|x-x_{0}\right\|
$$

and thus $\left\|x-x_{0}\right\| \leq\left\|z-z_{0}\right\|_{1} / c$. Using compactness, we may choose $z_{0} \in P x_{0}$ so that $\left\|y-z_{0}\right\|_{1}=\operatorname{dist}\left(y, P x_{0}\right)$. Now

$$
\left\|x-x_{0}\right\| \leq \frac{1}{c}\left\|z-z_{0}\right\|_{1} \leq \frac{1}{c}\left[\|z-y\|_{1}+\operatorname{dist}\left(y, P x_{0}\right)\right]
$$

and since $z$ is an arbitrary element of $P x$,

$$
\left\|x-x_{0}\right\| \leq \frac{1}{c}\left[\operatorname{dist}(y, P x)+\operatorname{dist}\left(y, P x_{0}\right)\right] \leq \frac{2 r}{c}=\varepsilon .
$$

and (3.2) is established

Define $D=\left\{x \in B\left(x_{0} ; \varepsilon\right): \operatorname{dist}(y, P x) \leq r\right\}$ and let $d=$ $\operatorname{dist}(y, P(D))$. It suffices to show $d=0$, for then there exists a sequence $\left\{x_{n}\right\} \subseteq D$ and a sequence $\left\{y_{n}\right\}$ converging to $y$ where, for each $n, y_{n} \in P x_{n}$. By (3.1), $\left\{x_{n}\right\}$ is Cauchy and thus converges to some $x_{\infty} \in D$; since $P$ is locally lipschitzian it follows easily that $y \in P x_{\infty}$.

Assume $d>0$ and fix $x \in D$. Choose $\tilde{z} \in P x$ so that $\|y-\tilde{z}\|_{1}=$ $\operatorname{dist}(y, P x)$. Since $\tilde{\phi}(X)$ is dense; we may also choose $h \in X,\|h\| \geq 1$ with

$$
\|\tilde{\phi}(h)-\| y-\tilde{z}\left\|_{1}^{-1} J(y-\tilde{z})\right\|_{1} \leq \frac{c}{2 L} .
$$

Let $x_{t}=x+t h$ and let $t>0$ be sufficiently small so that (3.1) implies

$$
\left(z_{t}-\tilde{z}, \tilde{\phi}\left(x_{t}-x\right)\right) \geq c\left\|x_{t}-x\right\|^{2}
$$


for all $z_{t} \in P x_{t}$. This, in turn, yields

$$
\left(z_{t}-\tilde{z}, \tilde{\phi}(h)\right) \geq c t\|h\|^{2} \geq c\left\|x_{t}-x\right\|
$$

for all $z_{t} \in P x_{t}$. Using compactness once again, select $z_{t} \in P x_{t}$ so that $\left\|z_{t}-\tilde{z}\right\|_{1} \leq L\left\|x_{t}-x\right\|$ and observe for this particular point

$$
\left(z_{t}-\tilde{z}, \tilde{\phi}(h)\right) \geq \frac{c}{L}\left\|z_{t}-\tilde{z}\right\|_{1} .
$$

Combining the above inequality with (3.3) we have

$$
\begin{aligned}
&\left(z_{t}-\tilde{z}, J(y-\tilde{z})\right) \\
&=\left(z_{t}-\tilde{z},\|y-\tilde{z}\|_{1} \tilde{\phi}(h)-\|y-\tilde{z}\|_{1} \tilde{\phi}(h)+J(y-\tilde{z})\right) \\
&=\left(z_{t}-\tilde{z},\|y-\tilde{z}\|_{1} \tilde{\phi}(h)\right) \\
&-\|y-\tilde{z}\|_{1}\left(z_{t}-\tilde{z}, \tilde{\phi}(h)+\|y-\tilde{z}\|_{1}^{-1} J(y-\tilde{z})\right) \\
& \geq \frac{c}{L}\|y-\tilde{z}\|_{1}\left\|z_{t}-\tilde{z}\right\|_{1}-\frac{c}{2 L}\|y-\tilde{z}\|_{1}\left\|z_{t}-\tilde{z}\right\|_{1} \\
&= \frac{c}{2 L}\|y-\tilde{z}\|_{1}\left\|z_{t}-\tilde{z}\right\|_{1} .
\end{aligned}
$$

Using the well-known fact that $J$ is the subgradient of $\frac{1}{2}\|\cdot\|_{1}^{2}$ (i.e., $\|u\|_{1}^{2} \leq\|v\|_{1}^{2}-2(v-u, J(u))$ for all $\left.u, v \in Y\right)$, we use the last inequality to estimate $\left\|y-z_{t}\right\|_{1}$ :

$$
\begin{aligned}
\left\|y-z_{t}\right\|_{1}^{2} \leq & \|y-\tilde{z}\|_{1}^{2}-2\left(z_{t}-\tilde{z}, J\left(y-z_{t}\right)\right) \\
= & \|y-\tilde{z}\|_{1}^{2}-2\left(z_{t}-\tilde{z}, J(y-\tilde{z})\right) \\
& +2\left(z_{t}-\tilde{z}, J(y-\tilde{z})-J\left(y-z_{t}\right)\right) \\
\leq & \|y-\tilde{z}\|_{1}^{2}-\frac{c}{L}\|y-\tilde{z}\|_{1}\left\|z_{t}-\tilde{z}\right\|_{1} \\
& +2\left\|z_{t}-\tilde{z}\right\|_{1}\left\|J(y-\tilde{z})-J\left(y-z_{t}\right)\right\|_{1} .
\end{aligned}
$$

Since $P$ is locally lipschitzian and $J$ is norm-to-norm continuous, we can choose $t>0$ sufficiently small so that

$$
\left\|J(y-\tilde{z})-J\left(y-z_{t}\right)\right\|_{1} \leq \frac{c d}{4 L}
$$

and since $\tilde{z} \in P x,\|y-\tilde{z}\|_{1} \geq \operatorname{dist}(y, P(D))=d$. Combining (3.4) and (3.5), we obtain

$$
\left\|y-z_{t}\right\|_{1}^{2} \leq\|y-\tilde{z}\|_{1}^{2}-\frac{c d}{L}\left\|z_{t}-\tilde{z}\right\|_{1}+\frac{c d}{2 L}\left\|z_{t}-\tilde{z}\right\|_{1},
$$


which when rewritten yields

$$
\begin{aligned}
\frac{c d}{2 L}\left\|z_{t}-\tilde{z}\right\|_{1} & \leq\|y-\tilde{z}\|_{1}^{2}-\left\|y-z_{t}\right\|_{1}^{2} \\
& \leq\left[\operatorname{dist}(y, P(x)]^{2}-\left[\operatorname{dist}\left(y, P x_{t}\right)\right]^{2} .\right.
\end{aligned}
$$

Note, in particular, this says $\left\|y-z_{t}\right\|_{1} \leq\|y-\tilde{z}\|_{1}$ and hence $\operatorname{dist}\left(y, P x_{t}\right) \leq r$.

We now construct a function $g$ on $D$ : let $x \in D$ and choose $t>0$ sufficiently small so that (3.6) holds and $\left\|x_{t}-x\right\| \leq \varepsilon$. Set $g(x)=x_{t}$; (3.2) then implies $g: D \rightarrow D$. Moreover, since $g(x) \in D$, we can combine (3.1) and (3.6) to obtain

$$
\frac{c^{2} d}{2 L}\|x-g(x)\| \leq[\operatorname{dist}(y, P x)]^{2}-\left[\operatorname{dist}\left(y, P x_{t}\right)\right]^{2} .
$$

Since the mapping $\psi_{0}: x \rightarrow \operatorname{dist}(y, P x)$ is 1.s.c., an application of Theorem $\mathrm{C}$ with $\psi=2 L \psi_{0}^{2} / c^{2} d$ provides a contradiction to the assumption that $d>0$. Thus $y \in P(U)$ and $P(U)$ is open, finishing the proof of Theorem 2.1.

We note that if $A$ is a closed, convex, bounded subset of a reflexive space $Y$, then $A$ is proximinal; i.e., given any $y \in Y$, there exists $a \in A$ with $\|y-a\|=\operatorname{dist}(y, A)$. Moreover, if $P: X \rightarrow \Re(Y)$ is a mapping and $u, v \in X$ are such that $H(P u, P v) \leq L\|u-v\|$, given $z \in P u$ and $L_{0}>L$ the remarks prior to the proof of Theorem 2.1 allow us to choose $w \in P v$ with $\|w-z\| \leq L_{0}\|u-v\|$.

Proof of Theorem 2.2. Since reflexive spaces are clearly WCG, Proposition $\mathrm{D}$ guarantees the existence of an equivalent norm $\|\cdot\|_{1}$ on $Y$ so that $\left(Y,\|\cdot\|_{1}\right)$ has Fréchet differentiable norm and $\left(Y,\|\cdot\|_{1}\right)^{*}$ is strictly convex; moreover, for each $x \in X, P x$ is a closed, convex, bounded subset of the reflexive space $\left(Y,\|\cdot\|_{1}\right)$. Let $L$ be the local Lipschitz constant of the mapping $P: X \rightarrow \Re(Y)$ and fix $L_{0}>L$. By the preceding comments, we may now duplicate the proof of Theorem 2.1 , replacing $L$ by $L_{0}$, to establish Theorem 2.2.

The proof of Theorem 2.3 is, as was the case with Theorem 2.2, essentially a minor variation of the proof of Theorem 2.1 . We employ the same notation as previously.

Proof of Theorem 2.3. Let $\|\cdot\|_{1}$ be the norm on $Y$ so that $J$ : $\left(Y,\|\cdot\|_{1}\right) \rightarrow\left(Y,\|\cdot\|_{1}\right)^{*}$ is lipschitzian on bounded sets. Let $U \subseteq X$ be open, fix $x_{0} \in U$ and choose $\varepsilon>0$ so that $B\left(x_{0}, 2 \varepsilon\right) \subseteq U$ and (3.1) holds 
on $B\left(x_{0}, 2 \varepsilon\right)$. Let $r=c \varepsilon / 2$ and let $y \in V\left(P x_{0} ; r\right)$. Note that (3.2) is still valid and that it will suffice to show $d=\operatorname{dist}(y, P(D))=0$ where $D=$ $\left\{x \in B\left(x_{0} ; \varepsilon\right): \operatorname{dist}(y, P x) \leq r\right\}$.

Fix $x \in D$. Choose $\tilde{z} \in P x$ so that $\|y-\tilde{z}\|_{1}=\operatorname{dist}(y, P x)$ and, since $\phi(X)=Y^{*}$ implies $\tilde{\phi}(X)=Y^{*}$, we may also choose $h \in X$ with $\tilde{\phi}(h)=$ $J(y-\tilde{z})$. Let $x_{t}=x+t h$. Since $\|y-\tilde{z}\|_{1} \leq\|h\|,(3.1)$ implies

$$
\left(z_{t}-\tilde{z}, J(y-\tilde{z})\right) \geq c\left\|x_{t}-x\right\|\|y-\tilde{z}\|_{1}
$$

for all $z_{t} \in P x_{t}$ whenever $t>0$ is sufficiently small. Let $z_{t} \in P x_{t}$ and apply (3.7) to get

$$
\begin{aligned}
{\left[\operatorname{dist}\left(y, P x_{t}\right)\right]^{2} \leq } & \left\|y-z_{t}\right\|_{1}^{2} \leq\|y-\tilde{z}\|_{1}^{2}-2\left(z_{t}-\tilde{z}, J\left(y-z_{t}\right)\right) \\
= & \|y-\tilde{z}\|_{1}^{2}-2\left(z_{t}-\tilde{z}, J(y-\tilde{z})\right) \\
& +2\left(z_{t}-\tilde{z}, J(y-\tilde{z})-J\left(y-z_{t}\right)\right) \\
\leq & \|y-\tilde{z}\|_{1}^{2}-2 c\left\|x_{t}-x\right\|\|y-\tilde{z}\|_{1} \\
& +2\left\|z_{t}-\tilde{z}\right\|_{1}\left\|J(y-\tilde{z})-J\left(y-z_{t}\right)\right\|_{1} .
\end{aligned}
$$

Let $z_{t} \in P x_{t}$ be choosen so that $\left\|z_{t}-\tilde{z}\right\|_{1}=\operatorname{dist}\left(\tilde{z}, P x_{t}\right)$ and let $M$ be the Lipschitz constant of $J$ on $B(0 ; 2 r)$. Then from the above, assuming $d>0$, we have

$$
\left[\operatorname{dist}\left(y, P x_{t}\right)\right]^{2} \leq[\operatorname{dist}(y, P x)]^{2}-2 c d\left\|x_{t}-x\right\|+2 M\left[\operatorname{dist}\left(\tilde{z}, P x_{t}\right)\right]^{2},
$$

provided that $\operatorname{dist}\left(\tilde{z}, P x_{t}\right) \leq 2 r$, which, by (2.5), is true for all $t>0$ sufficiently small. In fact, (2.5) implies

$$
\left[\operatorname{dist}\left(\tilde{z}, P x_{t}\right)\right]^{2} \leq \varepsilon(t)\left\|x_{t}-x\right\|
$$

where $\varepsilon(t) \rightarrow 0$ as $t \rightarrow 0$. Thus, for $t$ near zero, $\varepsilon(t) \leq c d / 2 M$ and

$$
\left[\operatorname{dist}\left(y, P x_{t}\right)\right]^{2} \leq[\operatorname{dist}(y, P x)]^{2}-c d\left\|x_{t}-x\right\| \text {. }
$$

(3.2) again implies $x_{t} \in D$ and hence we once more obtain a contradiction via Theorem $\mathrm{C}$.

We conclude by comparing our definition of locally strongly $\phi$-accretive with that introduced by Kirk [13]. To ease this discussion, consider the case where $P: X \rightarrow Y$. In [13], and subsequently in [15], $P: X \rightarrow Y$ is said to be locally strong $\phi$-accretive if, for each $y \in Y$ and $r>0$, there is a $c>0$ such that

(2.1) $\quad$ If $\|P x-y\| \leq r$, then, for all $u \in X$ sufficiently near $x$

$$
(P u-P x, \phi(u-x)) \leq c\|x-u\|^{2} \text {. }
$$


Thus our definition varies from that employed by Kirk in two instances. First he assumes that inequality $(2.1)^{\prime}$ holds only for pairs of the form $(u, x)$ in some neighborhood of $x$, we assume the stronger condition that (2.1) holds for all pairs of points in the neighborhood. Our stronger condition enables us to show $P$ is locally closed; this is essential to the proofs of Theorems 2.1 and 2.2. On the other hand, Kirk's constant $c$ depends on $y$ and holds for any $x$ with $\|P x-y\| \leq r$; in (2.1) we impose a weaker condition that $c$ need only depend on $x$. Since $c=c(x)$, we are able to derive Browder's result [1, Theorem 4.11] as a corollary (Corollaries 2.2 and 2.3).

As mentioned earlier, Corollary 2.1 (and the corresponding result for Theorems 2.2 and 2.3) remain valid under Kirk's definition (see [9] for the set-valued version). To see this we again modify the proof of Theorem 2.1. Suppose $P(X)$ is closed, fix $x_{0} \in X$ and choose $\varepsilon_{1}$ so that $P$ is lipschitzian on $B\left(x_{0} ; 2 \varepsilon_{1}\right)$. Select $c>0$ and $\varepsilon_{2}>0$ so that

$$
\begin{aligned}
& \text { if } z \in P(X) \cap V\left(P x_{0} ; 2 L \varepsilon_{1}\right) \text { and } x \in P^{-1}(z), \text { then if } \\
& \|u-x\| \leq 2 \varepsilon_{2}(z-w, \tilde{\phi}(x-u)) \geq c\|x-u\|^{2} \text { for all } w \in P u .
\end{aligned}
$$

Set $\varepsilon=\min \left\{\varepsilon_{1}, \varepsilon_{2}\right\}$ and $r=\min \{c \varepsilon / 2, L \varepsilon\}$ where $L$ is the local Lipschitz constant of $P$. Let $y \in V\left(P x_{0} ; r\right)$; we need only show $y \in P(X)$. Claim (3.2) remains valid, for if $z \in P x$,

$$
\operatorname{dist}\left(z, P x_{0}\right) \leq H\left(P x, P x_{0}\right) \leq L\left\|x-x_{0}\right\| \leq 2 L \varepsilon
$$

and so by $(3.1)^{\prime}$

$$
\begin{aligned}
c\left\|x-x_{0}\right\|^{2} & \leq\left(z-z_{0}, \tilde{\phi}\left(x-x_{0}\right)\right) \\
& \leq\left\|z-z_{0}\right\|_{1}\left\|x-x_{0}\right\| \text { for all } z_{0} \in P x_{0} .
\end{aligned}
$$

Let $d=\operatorname{dist}(y, P(X))$. If $d>0$, the construction in the proof of Theorem 2.1 remains intact and again provides a contradiction to Caristi's Theorem. Thus $d=0$ and $y \in P(X)$.

\section{REFERENCES}

1. F. E. Browder, Nonlinear Operators and Nonlinear Equations of Evolution in Banach Spaces, Proc. Symp. Pure Math. Vol. 18, part 2, Amer. Math. Soc., Providence, RI, 1976. 2. _ Normal solvability and existence theorems for nonlinear mappings in Banach spaces, in Problems in Nonlinear Analysis, pp. 19-35, Edizioni Cremonese, Roma, Italia, 1971.

3. Normal solvability for nonlinear mappings and the geometry of Banach spaces, in Problems in Nonlinear Analysis, pp. 37-66, Edizioni Cremonese, Roma, Italia, 1971.

4. , Normal solvability and $\phi$-accretive mappings of Banach spaces, Bull. Amer. Math. Soc., 78 (1972), 186-192.

5. J. Caristi, Fixed point theorems for mappings satisfying inwardness conditions, Trans. Amer. Math. Soc., 215 (1976), 241-251. 
6. M. M. Day, Normed Linear Spaces, 3rd Ed., Ergebnisse der Mathematik und ihrer Grenzgebiete, Band 21, Springer-Verlag, New York, 1973.

7. M. Deimling, Zeros of accretive operators, Manuscripta Math., 13 (1974), 365-374.

8. J. Diestel, Geometry of Banach Spaces-Selected Topics, Lecture Notes in Mathematics no. 485, Springer-Verlag, New York, 1975.

9. D. J. Downing, Surjectivity results for $\phi$-accretive set-valued mappings, Pacific J. Math., 77 (1978), 381-388.

10. I. Ekeland, Sur les problemes variationnels, Compte Rendus Acad. Soc. Paris, 275 (1972), 1057-1059.

11. P. Enflo, Banach spaces which can be given an equivalent uniformly convex norm, Israel J. Math., 13 (1972), 281-288.

12. K. John and V. Zizler, Smoothness and its equivalents in weakly compactly generated Banach spaces, J. Functional Analysis, 15 (1974), 1-11.

13. W. A. Kirk, Caristi's fixed point theorem and the theory of normal solvability, Proc. Conf. on Fixed Point Theory and its Applications, Dalhoousie University, June 1975, Academic Press, New York, 1976, pp. 109-120.

14. W. V. Petryshyn, Nonlinear equations involving non-compact operators, Symp. on Nonlinear Functional Analysis, 1968, Proc. Symp. Pure Math. Vol. 18, part 1, Amer. Math. Soc., Providence, RI, 1970, pp. 206-233.

15. W. O. Ray, Phi-accretive operators and Ekeland's theorem, to appear in the Journal of Math. Anal. and Appl.

16. W. O. Ray and A. Walker, Mapping theorems for Gateaux différentiable and accretive operators, preprint.

17. V. L. Smul'yan, Sur les topologies differentes dans l'espace de Banach, C.R. (Dokl.) Acad. Sci. URSS, 23 (1939), 331-334.

18. , Sur la derivabilite de la norm dans l'espace de Banach, C.R. (Dokl.) Acad.

Sci. URSS, 27 (1940), 643-648.

19. S. Troyanski, On locally uniformly convex and differentiable norms in certain nonseparable Banach spaces, Studia Math., 37 (1971), 173-180.

Received September 23, 1981. Part of this work was carried out while the first author was a Visiting Scholar at the University of Oklahoma. Research of the second author was supported in part by a grant from the University of Oklahoma Foundation.

OAKLAND UNIVERSITY

ROCHESTER, MI 48063

AND

UNIVERSITY OF OKLAHOMA

NoRMAN, OK 73019 



\section{PACIFIC JOURNAL OF MATHEMATICS EDITORS}

DONALD BABBITT (Managing Editor)

University of California

Los Angeles, CA 90024

Hugo Rossi

University of Utah

Salt Lake City, UT 84112

C. C. Moore and Arthur Ogus

University of California

Berkeley, CA 94720
J. DugunduI

Department of Mathematics

University of Southern California

Los Angeles, CA 90089-1113

R. FinN and H. SAmelson

Stanford University

Stanford, CA 94305

\section{ASSOCIATE EDITORS}
R. ARENS
E. F. BECKENBACH
B. H. NeUmaNN
F. WolF
K. YoshidA (1906-1982)

\section{SUPPORTING INSTITUTIONS}

UNIVERSITY OF ARIZONA

UNIVERSITY OF BRITISH COLUMBIA

CALIFORNIA INSTITUTE OF TECHNOLOGY

UNIVERSITY OF CALIFORNIA

MONTANA STATE UNIVERSITY

UNIVERSITY OF NEVADA, RENO

NEW MEXICO STATE UNIVERSITY

OREGON STATE UNIVERSITY
UNIVERSITY OF OREGON

UNIVERSITY OF SOUTHERN CALIFORNIA

STANFORD UNIVERSITY

UNIVERSITY OF HAWAII

UNIVERSITY OF TOKYO

UNIVERSITY OF UTAH

WASHINGTON STATE UNIVERSITY

UNIVERSITY OF WASHINGTON 


\section{Pacific Journal of Mathematics}

\section{Vol. 106, No. $1 \quad$ November, 1983}

John Ballard, Clifford's theorem for algebraic groups and Lie algebras . . . . . 1

Philip Richard Bartick, II and Edwin Duda, Finite-to-one open mappings

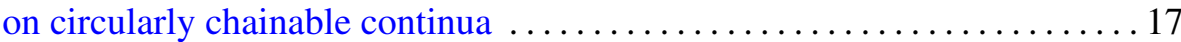

Frank Hayne Beatrous, Jr., $H^{\infty}$-interpolation from a subset of the

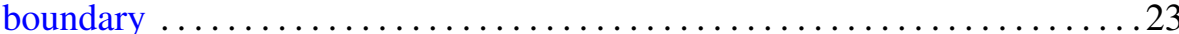

Sterling K. Berberian, Expectations in semifinite algebras ............ 33

Heron S. Collins and Wolfgang Ruess, Weak compactness in spaces of compact operators and of vector-valued functions $\ldots \ldots \ldots \ldots \ldots \ldots . \ldots 45$

David Downing and William O. Ray, Renorming and the theory of

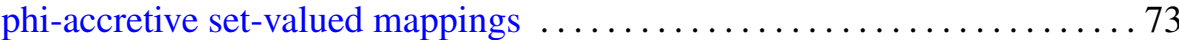

Harvey Charles Greenwald, On the theory of homogeneous Lipschitz

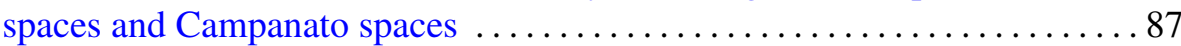

Irwen Valle Guadalupe and Lucio Ladislao Rodriguez, Normal curvature

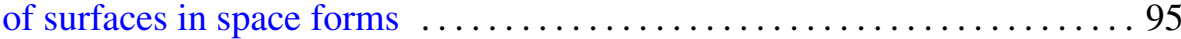

Boju Jiang, On the computations of the Nielsen number ............... 105

William H. Julian, Ray Mines, III and Fred Richman, Alexander

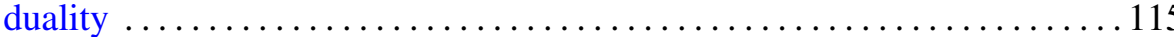

Midori Kobayashi, The connected component of the idèle class group of an

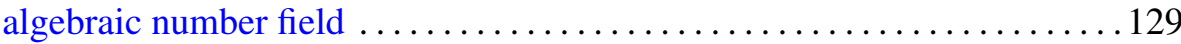

Ib Henning Madsen, Charles B. Thomas and C. Terence C. (Charles)

(Clegg) Wall, Topological spherical space form problem. III.

Dimensional bounds and smoothing

A. Sapounakis, The existence of strong liftings for totally ordered measure

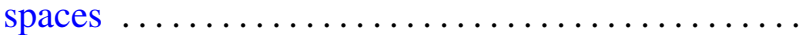

Gary Seitz, The root subgroups for maximal tori in finite groups of Lie type

Laurent Siebenmann and James M. Van Buskirk, Construction of irreducible homology 3-spheres with orientation reversing involution 